\title{
MVZ werden hoffähig
}

— Die Akzeptanz von Medizinischen Versorgungszentren (MVZ) steigt zusehends. Das betonte Gesundheitsstaatssekretär Lutz Stroppe beim Praktikerkongress des Bundesverbands MVZ (BMVZ) am 16.09.2015 in Berlin. Auch in der Gesundheitspolitik der Union habe sich ein Sinneswandel vollzogen. Man habe gesehen, dass MVZ neue Möglichkeiten der Patientenversorgung schaffen. Die Koalition habe mit dem Versorgungsstärkungsgesetz versucht, die Möglichkeiten für MVZ zu erweitern und die Voraussetzungen für eine weitere positive Entwicklung zu schaffen. Als „wichtige Weiterentwicklung" bewertete er die Möglichkeit zur Gründung fachgleicher MVZ. „Ein Schritt, um das Instrument weiter in die Fläche zu bringen“ seien die Möglichkeiten für Kommunen, MVZ zu gründen.

Einen gewandelten Blickwinkel auf MVZ nimmt auch der frühere Chef der Kassenärztlichen Bundesvereinigung (KBV) und jetzige Ehrenvorsitzende des Spitzenver- bands der Fachärzte Dr. Andreas Köhler ein. Die Zunahme von MVZ und angestellten Ärzten in der ambulanten Medizin bezeichnete Köhler als "dramatische Veränderung".. „Dieser Trend ist spürbar, und dem muss man sich stellen", sagte der Ex-Funktionär mit Seitenhieb auf das KV-System. Köhler forderte die ärztliche Selbstverwaltung auf, ein neues Verständnis von ärztlicher Freiberuflichkeit zu entwickeln. „Wir dürfen das nicht mehr mit wirtschaftlicher Selbstständigkeit gleichsetzen", sagte Köhler.

Seine "ganz persönlichen" Appelle an die verfasste Ärzteschaft: „Wir müssen die Strukturen für die nachwachsende Generation aufbauen. Dazu gehört auch, dass wir MVZ als eine Form der Kooperation akzeptieren müssen. So weit ist das KV-System noch nicht." Den KVen empfahl er, MVZ besser zu integrieren. „Sie werden zu viele, als dass ich nicht Sorge hätte, dass sie sich irgendwann eine andere Interessenvertretung suchen", so Köhler. Angela Mißlbeck

Medizinklimaindex

Ärzte beurteilen ihre Lage optimistisch

— Der Medizinklimaindex (MKI) der Stiftung Gesundheit kletterte im Frühjahr 2015 zum ersten Mal für Haus- wie auch Fachärzte ins Plus. Die beiden Gruppen wurden erstmals getrennt be-
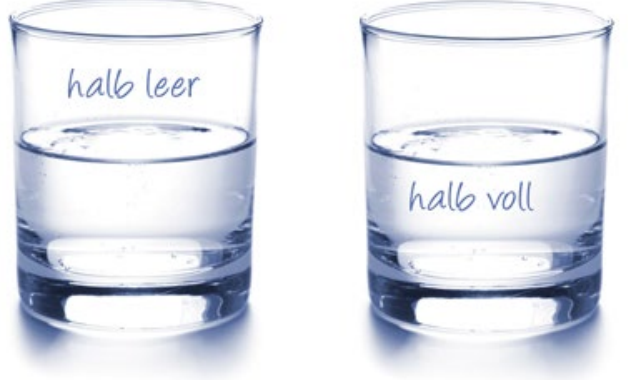

Haus- und Fachärzte schauen positiv in ihre wirtschaftliche Zukunft. trachtet. Die Ärzte schätzen ihre aktuelle und zukünftige wirtschaftliche Situation optimistischer ein als bisher: Fachärzte mit 6,3 Punkten, Hausärzte mit 6,1 Punkten. Im Herbst 2014 lag der gemeinsame MKI von Haus- und Fachärzten noch bei -7,4. Auch die anderen Berufsgruppen verbessern ihr Stimmungsbild. Der MKI der Psychologischen Psychotherapeuten kommt mit 3,2 Punkten zurück aus dem Minus (Herbst 2014: -2,6). Der Gesamtwert der Haus-, Fach- und Zahnärzte sowie der Psychologischen Psychotherapeuten ist mit 6,2 Punkten (Herbst 2014: -3,6) der höchste seit Beginn der Erhebung im Jahr 2006. Zudem liegt der MKI erstmals für alle Berufsgruppen im positiven Bereich. Er wird im Auftrag der Stiftung Gesundheit von der Gesellschaft für Gesundheitsmarktanalyse mbH erhoben. Brigitta Schneider

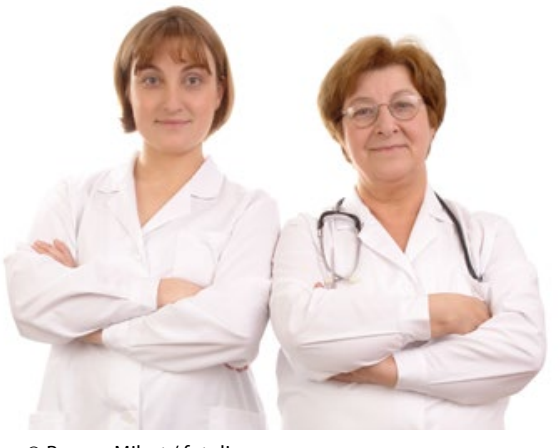

(c) Roman Milert / fotolia.com

\section{Eigene Praxis}

\section{Deutlich weniger Verdienst für Ärztinnen}

— Das Zentralinstitut für die kassenärztliche Versorgung ( $\mathrm{Zi}$ ) hat in seinem aktuellen Praxis-Panel ermittelt, dass nicht nur angestellte, sondern auch selbstständig tätige Ärztinnen beim Einkommen ihren männlichen Kollegen hinterherhinken. Während die Praxisinhaber über alle Fachgruppen hinweg in der Erhebungswelle 2013 (Basis: Praxisdaten von 2009-2011) einen Jahresüberschuss von im Schnitt $168.800 €$ erwirtschafteten, blieben ihren Kolleginnen nur $104.600 €$. Dabei sind die Unterschiede innerhalb der einzelnen Fachgruppen größtenteils zwar geringer, aber auf einen gleich hohen oder höheren Jahresüberschuss kommen niedergelassene Ärztinnen in keinem Fachgebiet.

Bei den Hausärzten beträgt die Differenz zwischen Praxisinhaber (162.600 $€$ ) und -inhaberin (119.900 €) beim Jahresüberschuss $42.700 €$. Jedoch zeigt sich, dass Ärztinnen über alle Fachbereiche hinweg mit 2.116 Stunden eine geringere Jahresarbeitszeit hatten als ihre Kollegen mit 2.520 Stunden. Entsprechend lag auch der Jahresüberschuss je Inhaberstunde bei Ärzten höher $(66,97 €)$ als bei Ärztinnen $(49,47 €)$. Zum Teil mag der Unterschied daran liegen, dass Ärztinnen mehr Zeit für ihre Patienten aufwenden und weniger Patienten behandeln. Sie kommen auf 38,3 Minuten je Patient und 763 Patienten, Ärzte nur auf 31,1 Minuten, aber 1.138 Patienten. Zudem behandeln Praxisinhaberinnen (Ausnahme: fachärztlicher Bereich I mit Augenheilkunde, Gynäkologie und Dermatologie) weniger Privatpatienten und sind stärker von GKV-Honoraren abhängig. Doch auch hier liegen ihre Einnahmen je GKV-Patient $(56,08 €)$ unter denen der Praxisinhaber $(60,58 €)$. Das Zi vermutet, dass Praxisinhaber mehr Leistungen je Fall abrechnen. Denn beim Aufwand je GKV-Patient liegen die Ärztinnen $(29,99 €)$ deutlich unter den Ärzten $(35,66 €)$. Rebecca Höhl 